\title{
Multiparametric Analysis of Permeability and ADC Histogram Metrics for Classification of Pediatric Brain Tumors by Tumor Grade
}

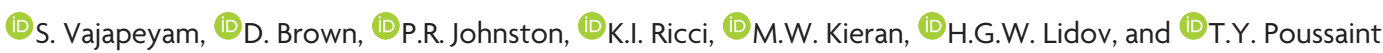

\begin{abstract}
BACKGROUND AND PURPOSE: Accurate tumor grading is essential for treatment planning of pediatric brain tumors. We hypothesized that multiparametric analyses of a combination of permeability metrics and ADC histogram metrics would differentiate high- and low-grade tumors with high accuracy.
\end{abstract}

MATERIALS AND METHODS: DTI and dynamic contrast-enhanced MR imaging using T1-mapping with flip angles of $2^{\circ}, 5^{\circ}, 10^{\circ}$, and $15^{\circ}$, followed by a $0.1-\mathrm{mmol} / \mathrm{kg}$ body weight gadolinium-based bolus was performed on all patients in addition to standard MR imaging. Permeability data were processed and transfer constant from the blood plasma into the extracellular extravascular space, rate constant from the extracellular extravascular space back into blood plasma, extravascular extracellular volume fraction, and fractional blood plasma volume were calculated from 3D tumor volumes. Apparent diffusion coefficient histogram metrics were calculated for 3 separate tumor volumes derived from T2-FLAIR sequences, T1 contrast-enhanced sequences, and permeability maps, respectively.

RESULTS: Results from 41 patients (0.3-16.76 years of age; mean, 6.22 years) with newly diagnosed contrast-enhancing brain tumors (16 low-grade; 25 high-grade) were included in the institutional review board-approved retrospective analysis. Wilcoxon tests showed a higher transfer constant from blood plasma into extracellular extravascular space and rate constant from extracellular extravascular space back into blood plasma, and lower extracellular extravascular volume fraction $(P<.001)$ in high-grade tumors. The mean ADCs of FLAIR and enhancing tumor volumes were significantly lower in high-grade tumors $(P<.001)$. ROC analysis showed that a combination of extravascular volume fraction and mean ADC of FLAIR volume differentiated high- and low-grade tumors with high accuracy (area under receiver operating characteristic curve $=0.918$ ).

CONCLUSIONS: ADC histogram metrics combined with permeability metrics differentiate low- and high-grade pediatric brain tumors with high accuracy.

ABBREVIATIONS: $A R O C=$ area under receiver operating characteristic curve; $D C E-M R I=$ dynamic contrast-enhanced $\mathrm{MR}$ imaging; $\mathrm{FL}=3 \mathrm{D}$ tumor volume from T2-FLAIR images; EES = extracellular extravascular space; $K_{\text {ep }}=$ rate constant from EES back into blood plasma; $K^{\text {trans }}=$ transfer constant from blood plasma into EES; $P G=3 D$ tumor volume from TI-contrast-enhanced images; $R O C=$ receiver operating characteristic; $v_{e}=$ extracellular extravascular volume fraction; $v_{p}=$ fractional blood plasma volume

P rimary brain tumors have an incidence rate of 5.67 per $100,000^{1}$ in children younger than 19 years of age and remain the most common cause of disease-related death in children. Accurate assessment of tumor grade is critical for diagnosis and treatment planning, especially with recent advances in

Received August 18, 2017; accepted after revision October 30.

From the Departments of Radiology (S.V., D.B., P.R.J., T.Y.P.) and Pathology (H.G.W.L.), Boston Children's Hospital, Boston, Massachusetts; Cancer Center (K.I.R.), Massachusetts General Hospital, Boston, Massachusetts; Division of Pediatric Oncology (M.W.K.), Dana-Farber Boston Children's Cancer and Blood Disorders Center, Boston, Massachusetts; and Harvard Medical School (S.V., M.W.K.,

H.G.W.L., T.Y.P.), Boston, Massachusetts.

Paper previously presented at: Annual Meeting of the American Society of Neuroradiology and the Foundation of the ASNR Symposium, April 22-27, 2017; Long Beach, California. genomic and molecular subtyping of tumors and targeted treatments for different subtypes. Conventional MR imaging remains the technique of choice for diagnosis and treatment; however, advanced imaging techniques such as perfusion and diffusion imaging have also proved useful for physiologic characterization of tumors.

Dynamic contrast-enhanced MR imaging (DCE-MRI) provides information on the vessel permeability and microvasculature in brain tumors and has been used extensively in adults in

Please address correspondence to Sridhar Vajapeyam, PhD, Department of Radiology, 300 Longwood Ave, Boston, MA 02115; e-mail:

sridhar.vajapeyam@childrens.harvard.edu

http://dx.doi.org/10.3174/ajnr.A5502 
tumor grading, ${ }^{2-5}$ but less frequently in children. ${ }^{6,7}$ DTI provides information about tumor cellularity and is useful for characterization of tissue, tumor grading, tumor response to treatment, and distinction of tissue types. ${ }^{8}$ ADC histogram analysis provides a comprehensive analysis of the volumetric diffusion characteristics of the tumor and has been used to predict survival in both adults ${ }^{9,10}$ and children. ${ }^{11}$

We retrospectively analyzed DCE-MR imaging and DTI data from children presenting with a brain mass during 3 years at Boston Children's Hospital and examined the permeability and ADC histogram metrics as predictors of tumor grade. We hypothesized that a combination of DCE-MR imaging and ADC histogram metrics would improve the accuracy of tumor grading on MR imaging.

\section{MATERIALS AND METHODS Subjects}

Children who presented with a brain mass from 2013 to 2017 who had DCE-MR imaging and DTI studies were included. The study was performed with the approval of the institutional review board of the Dana Farber Cancer Institute.

\section{MR Imaging Acquisition}

All MR imaging studies were performed on a 3T scanner (Skyra; Siemens, Erlangen, Germany). Standard MR imaging in all patients consisted of sagittal T1, axial T2, precontrast axial T2FLAIR, axial DTI $(\mathrm{TR}=6500 \mathrm{~ms}, \mathrm{TE}=88 \mathrm{~ms}$, slice thickness $=2$ $\mathrm{mm}, \mathrm{b}$-values of 0 and $1000 \mathrm{~s} / \mathrm{mm}^{2}, 30$ gradient directions), and postcontrast T1 images. All patients underwent a DCE-MR imaging protocol as follows: 1) variable flip angle echo-spoiled gradient-echo T1-mapping sequences using flip angles of $15^{\circ}, 10^{\circ}, 5^{\circ}$, and $2^{\circ} ; \mathrm{TR}=5$ seconds; $\mathrm{TE}=$ minimum; $\mathrm{FOV}=240 \mathrm{~mm}$; section thickness $=5 \mathrm{~mm}$; and 2) DCE-MR imaging sequence consisting of 50 phases, 7 seconds apart, flip angle $=15^{\circ}$, TR $=4$ seconds, $\mathrm{TE}=$ minimum. FOV, section thickness, and scan locations were identical to those in the T1-mapping sequences. A single bolus of gadobutrol (0.1-mL/kg body weight) was injected 20 seconds after the start of scanning at an injection rate of $2 \mathrm{~mL} / \mathrm{s}$.

\section{DCE-MR Imaging Postprocessing}

DCE-MR images were transferred to a VersaVue workstation (In vivo, Gainesville, Florida) for automated processing using OmniLook software. Voxelwise T1 maps were generated from the variable flip angle images, and the 2 -compartment Tofts model ${ }^{12}$ was used to generate voxelwise values of the pharmacokinetic parameters, namely the transfer constant from the blood plasma into the extracellular extravascular space $\left(K^{\text {trans }}\right)$, rate constant from extracellular extravascular space back into blood plasma $\left(K_{\mathrm{ep}}\right)$, extracellular extravascular volume fraction $\left(\mathrm{v}_{\mathrm{e}}\right)$, and fractional blood plasma volume $\left(\mathrm{v}_{\mathrm{p}}\right)$.

The model of Weinmann et $\mathrm{al}^{13}$ was used for blood plasma concentration. ROIs were drawn on each section of the tumor around contrast-enhancing portions of the tumor by an imaging data analyst or by a $\mathrm{PhD}$ scientist and verified by a Certificate of Added Qualification-certified neuroradiologist, and the mean (over voxels) and SDs of each of the variables were recorded. Only enhancing tumors were included in the analysis.

\section{ADC Histogram Analysis}

Due to their superior anatomic contrast, axial B0 images from the DTI sequence were registered to the axial T2-FLAIR images using the mutual information algorithm in FSL (http://www.fmrib.ox. ac.uk/fsl $)^{14}$ and the same transformation matrix used on the ADC maps to register them to the T2-FLAIR images. Axial postcontrast images were also separately registered to T2-FLAIR using the same methodology. 3D ROIs comprising the $3 \mathrm{D}$ tumor volume from T2-FLAIR images (FL) and the 3D tumor volume from T1contrast-enhanced images (PG) were automatically generated using the thresholding feature in Fiji (http://fiji.sc/), ${ }^{15}$ an Open Source (https://opensource.org/) distribution of Java modules along with ImageJ software (National Institutes of Health, Bethesda, Maryland). The ADC values of the voxels in the $3 \mathrm{D}$ tumor volume from T2-FLAIR images (FL), and PG ROIs were then used to generate the FL and PG ADC volumes, respectively. These volumes were thresholded using a uniform range of $600-2600 \times 10^{-6} \mathrm{~mm}^{2} / \mathrm{s}$ to automatically exclude cyst, necrosis, and hemorrhage. FL and PG ADC histograms were then generated using a uniform bin width of $7.8 \mathrm{~mm}^{2} / \mathrm{s}$ to facilitate comparison of shape descriptors of the histograms. Histogram metrics used for statistical analysis were the number of peaks (unimodal or bimodal), mean, SD, skewness, and kurtosis of these histograms. In the case of tumors showing bimodal ADC histograms, the lower peak was measured for mean and SD, and skewness and kurtosis were not recorded.

DCE-MR imaging and ADC histogram analyses were blinded to tumor grading results.

\section{Statistical Methods}

Low- and high-grade tumor groups were compared for permeability variables and ADC histogram metrics using the Wilcoxon test and $t$ test using the NPAR1WAY and $t$ test procedures of SAS (SAS Institute, Cary, North, Carolina). ${ }^{16}$ Corresponding to the $t$ test, estimates and $95 \%$ confidence intervals for the difference between means were calculated.

Receiver operating characteristic (ROC) curves and their areas (AROC) were estimated for highly statistically significant variables in the permeability set $\left(\mathrm{v}_{\mathrm{e}}, K^{\text {trans }}, K_{\mathrm{ep}}\right)$, ADC histogram set (FL_ADC_mean, PG_ADC_mean), and the 6 pairs of variables comprising 1 variable from each set. Focus was on the $v_{e}$ and FL_ADC_mean pair because these variables and their relationship were considered the most stable.

The ROC curve shows the trade-off between the true-positive rate (classifying the tumor of a patient with a high-grade tumor as high-grade) and false-positive rate (classifying the tumor of a patient with a low-grade tumor as high-grade). The AROC estimates the average true-positive rate over all possible false-positive rates and is also an estimate of the probability of correctly classifying a random pair of patients, 1 from the low-grade group and 1 from the high-grade group.

In addition to point estimates, Wald-based tests (corresponding to the null hypothesis that the true AROC is 0.5) and 95\% confidence intervals for the AROC were calculated. All estimates, confidence intervals, and tests were nonparametric and were calculated using the LOGISTIC procedure of SAS.

AJNR Am J Neuroradiol 39:552-57 Mar 2018 www.ajnr.org 
Table 1: Group comparisons: low- versus high-grade tumor

\begin{tabular}{|c|c|c|c|c|c|c|c|c|c|}
\hline Variable & $\begin{array}{l}\text { Low-Grade } \\
\text { (Mean) }\end{array}$ & $\begin{array}{l}\text { High-Grade } \\
\text { (Mean) }\end{array}$ & $\begin{array}{c}\text { Mean } \\
\text { Diff }\end{array}$ & $\begin{array}{c}\text { Mean } \\
\text { SE }\end{array}$ & $\begin{array}{l}\text { Lower } \\
95 \% \mathrm{CL}\end{array}$ & $\begin{array}{l}\text { Upper } \\
95 \% \mathrm{CL}\end{array}$ & $\begin{array}{l}P \text { Value } \\
\text { ( } T \text { Test) }\end{array}$ & $\begin{array}{c}\chi^{2} \\
\text { (Wilcoxon) }\end{array}$ & $\begin{array}{c}P \text { Value } \\
\text { (Wilcoxon) }\end{array}$ \\
\hline$K^{\text {trans }}\left(\min ^{-1}\right)$ & 0.14 & 1.50 & 1.36 & 0.45 & 0.46 & 2.27 & .004 & 16.72 & $<.001$ \\
\hline$K_{\mathrm{ep}}\left(\min ^{-1}\right)$ & 1.18 & 8.85 & 7.67 & 1.99 & 3.64 & 11.70 & $<.001$ & 18.98 & $<.001$ \\
\hline $\mathrm{v}_{\mathrm{e}}$ & 0.22 & 0.13 & -0.09 & 0.02 & -0.13 & -0.05 & $<.001$ & 13.41 & $<.001$ \\
\hline$v_{p}$ & 0.05 & 0.05 & 0.00 & 0.01 & -0.02 & 0.02 & .764 & 0.23 & .630 \\
\hline $\mathrm{FL}$ P ADC_mean $\left(\times 10^{-6} \mathrm{~mm}^{2} / \mathrm{s}\right)$ & 1458 & 1022 & -436 & 84.47 & -607 & -265 & $<.001$ & 15.65 & $<.001$ \\
\hline $\mathrm{FL} \_A D C \_S D\left(\times 10^{-6} \mathrm{~mm}^{2} / \mathrm{s}\right)$ & 336.8 & 285.0 & -51.8 & 36.01 & -125 & 21.06 & .158 & .56 & .454 \\
\hline FL_ADC_skew & 0.45 & 1.24 & 0.80 & 0.26 & 0.27 & 1.32 & .004 & 7.55 & .006 \\
\hline $\mathrm{FL}_{-} \mathrm{A} A \mathrm{C}_{-}$kurt & 1.28 & 1.97 & 0.70 & 0.73 & -0.79 & 2.18 & .347 & 1.58 & .208 \\
\hline PG_ADC_mean $\left(\times 10^{-6} \mathrm{~mm}^{2} / \mathrm{s}\right)$ & 1472 & 1066 & -406 & 100.7 & -610 & -203 & $<.001$ & 11.70 & $<.001$ \\
\hline PG_ADC_SD $\left(\times 10^{-6} \mathrm{~mm}^{2} / \mathrm{s}\right)$ & 261.0 & 308.4 & 47.46 & 40.47 & -34.4 & 129.3 & .248 & 1.93 & .165 \\
\hline PG_ADC_skew & 0.69 & 1.30 & 0.61 & 0.30 & 0.01 & 1.21 & .047 & 2.26 & .133 \\
\hline PG_ADC_kurt & 2.51 & 2.31 & -0.20 & 0.93 & -2.09 & 1.70 & .835 & 0.34 & .560 \\
\hline
\end{tabular}

Note:-Diff indicates difference; SE, standard error; CL, confidence limit; skew, skewness; kurt, kurtosis.

\section{RESULTS}

Sixty-three patients were identified. Six patients had final diagnoses that were not brain tumors, 10 had nonenhancing tumors and were excluded from DCE-MR imaging analysis, and 6 patients were excluded due to motion or susceptibility artifacts. The remaining 41 patients were included.

Twenty-three children were boys, and 18, girls (age range, $0.30-16.76$ years; median age, 4.76 years; mean age, 6.22 years). There were 31 infratentorial and 10 supratentorial tumors, and all except 2 were newly diagnosed.

Forty patients had enhancing biopsy-confirmed tumors, and 1 had a diffuse midline glioma located in the pons presumed to be high-grade. World Health Organization grades 1-2 were considered low-grade, whereas grades 3-4 were considered high-grade. Of these tumors, 16 were confirmed low-grade ( 7 pilocytic astrocytomas, 5 low-grade gliomas, 1 mature teratoma, 1 atypical meningioma, 1 low-grade ganglioglioma, and 1 low-grade mixed germ cell tumor). Twenty-five tumors were high-grade (12 medulloblastomas, 4 glioblastomas, 4 anaplastic ependymomas, and 1 each of atypical teratoid/rhabdoid tumor, embryonal tumor not otherwise specified, choroid plexus carcinoma, embryonal tumor with rhabdoid features, and diffuse midline glioma). Not all final diagnoses were given as the World Health Organization 2016 classification because some cases did not fit well into this classification at the time of diagnosis.

Molecular subtyping was successful in 11 of the 12 patients with medulloblastoma, yielding 3 sonic hedgehog/SHH-activated, 2 wingless/WNT-activated, and 6 classified as group 3 or 4 .

There was no statistically significant difference in mean ages between the low-grade (mean, 7.28 years; median, 5.31 years) and the high-grade cohort (mean, 5.54 years; median, 4.57 years) $(P=$ $.552)$.

Group comparisons of all permeability and ADC histogram variables are shown in Table 1. Of the pharmacokinetic variables analyzed, $K^{\text {trans }}$ and $K_{\mathrm{ep}}$ were significantly higher in high-grade tumors compared with low-grade tumors $(P<.001)$, and $\mathrm{v}_{\mathrm{e}}$ was significantly lower in the high-grade group $(P<.001)$.

Of the ADC histogram metrics analyzed, the mean ADC of the T2-FLAIR tumor volume (FL_ADC_mean) and the PG tumor volume (PG_ADC_mean) were significantly higher for low-grade tumors (Fig 1$)$ compared with high-grade tumors $(P<.001)$ (Fig 2). Low-grade tumors also showed a tendency for lower skewness for T2-FLAIR tumor volume (FL_ADC_skew, $P=$ .006). None of the other variables showed statistically significant group differences between low- and high-grade tumors. Six high-grade tumors showed bimodal T2-FLAIR ADC histograms, and 4 showed bimodal PG ADC histograms, while all the ADC histograms for low-grade tumors had just a single peak.

No group differences were found among the molecular subtypes in the medulloblastoma cohort $(P \geq .294)$.

ROC analysis of the 5 highly statistically significant variables ( $K^{\text {trans }}, K_{\text {ep }}, v_{\mathrm{e}}$, FL_ADC_mean, and PG_ADC_mean) showed that all displayed high sensitivity and specificity in discriminating low- and high-grade tumors, with AROC values above $0.82(P<$ .001) as seen in Table 2. AROC values for $\mathrm{v}_{\mathrm{e}}$ and FL_ADC_mean were 0.843 and 0.870 , respectively, while the AROC estimate for the 2-variable combination of these metrics was 0.918 (Fig 3). Focus was on this pair because the individual variables and their relationship were considered the most stable.

The Spearman correlation between $\mathrm{v}_{\mathrm{e}}$ and FL_ADC_mean across the 41 tumors was $0.49(P=.001)$. Correlation within the low- and high-grade groups was not significant.

\section{DISCUSSION}

Multiparametric imaging of pediatric brain tumors provides physiologic information beyond standard MR imaging. Koob et $\mathrm{al}^{7}$ recently reported increased accuracy in differentiating grades I and IV pediatric brain tumors using a combination of diffusion and DSC perfusion metrics. Similarly, Morana et al ${ }^{17}$ showed improved prognostic capability by combining DWI, arterial spinlabeling, and PET data in pediatric diffuse astrocytic tumors. Other studies have demonstrated the advantage of combinations of DWI, DSC perfusion, MR spectroscopy, and PET data in studying pediatric brain tumors. ${ }^{18-20}$ To our knowledge, this is the first study to demonstrate a multiparametric approach using DCE and ADC histogram metrics in pediatric brain tumors.

While DSC and arterial spin-labeling perfusion metrics have been reported for pediatric brain tumor grading, ${ }^{21-23}$ there are fewer studies using DCE-MR imaging. ${ }^{6}$ Our results show that among the DCE-MR imaging parameters, $K^{\text {trans }}, K_{\mathrm{ep}}$, and $\mathrm{v}_{\mathrm{e}}$ all successfully distinguished low- and high-grade tumors, and this result is consistent with our report from a smaller cohort. ${ }^{6}$ Highgrade tumors exhibited higher values of $K^{\text {trans }}$ and $K_{\text {ep }}$, indi- 


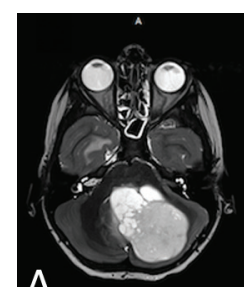

A

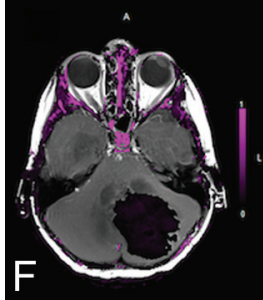

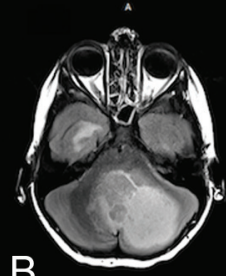

B

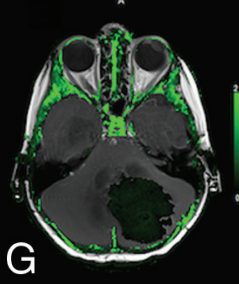

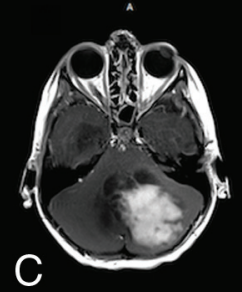

C

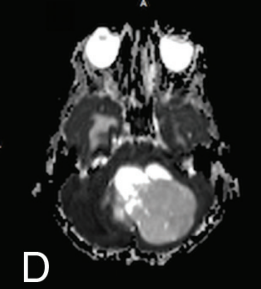

$\mathrm{D}$

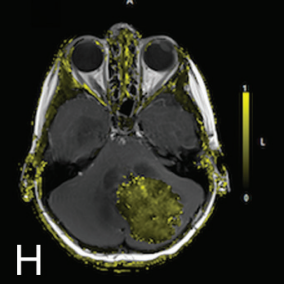

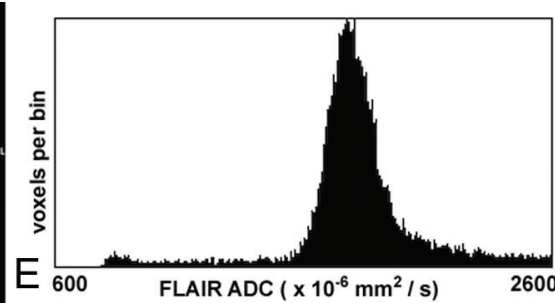

FIG 1. A 6-year-old boy with a low-grade pilocytic astrocytoma. The upper row shows the T2-weighted image $(A)$, T2-FLAIR image (B), postcontrast T1-weighted image $(C)$, ADC map $(D)$, and the ADC histogram of the FLAIR tumor volume $(E)$, respectively. The lower row shows the $K^{\text {trans }}$ $\operatorname{map}(F), K_{\mathrm{ep}} \operatorname{map}(G), \mathrm{v}_{\mathrm{e}} \operatorname{map}(H), \mathrm{v}_{\mathrm{p}}$ map $(I)$, and the $A D C$ histogram of the enhancing tumor volume $(l)$, respectively. The enhancing tumor volume was defined as the nodular enhancement of the solid component for pilocytic astrocytomas. Note the low $K^{\text {trans }}$ and $K_{\mathrm{ep}}$ and the high $v_{\mathrm{e}}$ values in this low-grade tumor. The mean ADC of the FLAIR tumor volume is $1821 \times 10^{-6} \mathrm{~mm}^{2} / \mathrm{s}$ and $1808 \times 10^{-6} \mathrm{~mm}^{2} / \mathrm{s}$ for the enhancing tumor volume.
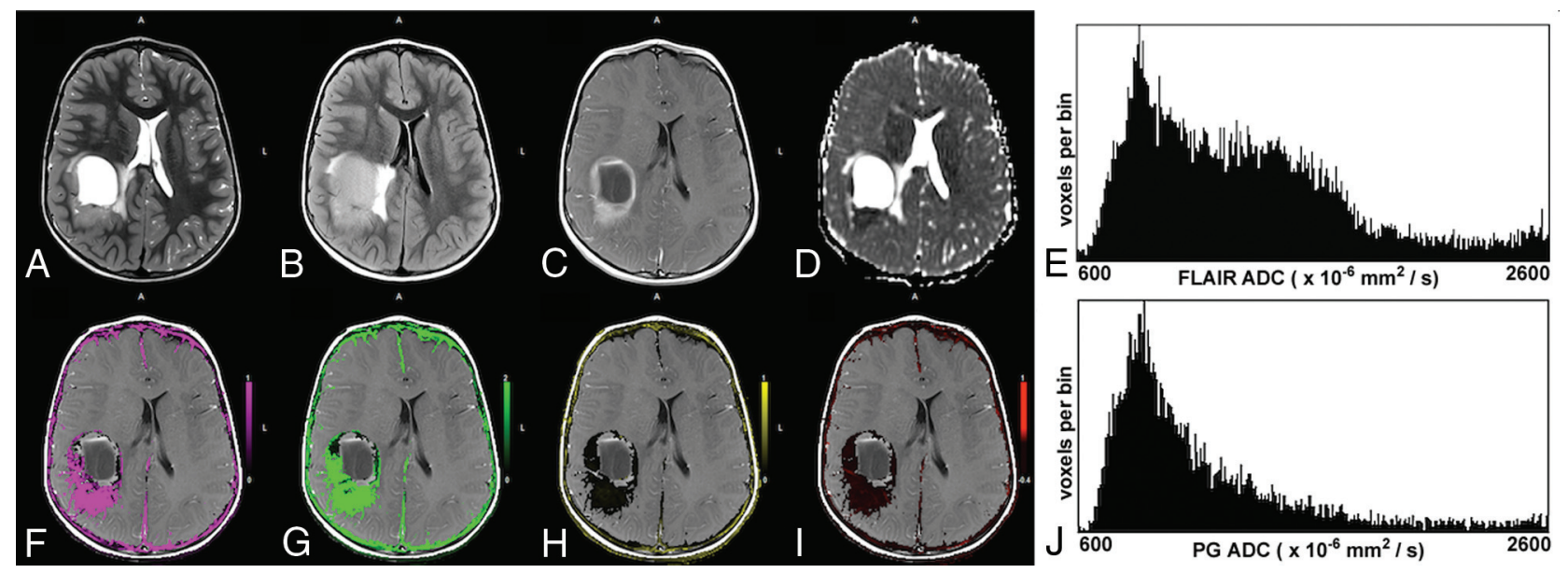

FIG 2. A 5-year-old boy with parietal anaplastic ependymoma. The upper row shows the T2-weighted image (A), T2-FLAIR image (B), postcontrast Tl-weighted image $(C), A D C$ map $(D)$, and the ADC histogram of the FLAIR tumor volume $(E)$, respectively. The lower row shows the $K^{\text {trans }}$ $\operatorname{map}(F), K_{\text {ep }} \operatorname{map}(G), v_{\mathrm{e}}$ map $(H), v_{\mathrm{p}}$ map $(I)$, and the $A D C$ histogram of the enhancing tumor volume $(l)$, respectively. Note the high $K^{\text {trans }}$ and $K_{\text {ep }}$ and the lower $v_{e}$ values in this high-grade tumor. The histogram of the FLAIR tumor volume is bimodal. The mean ADC of the lower peak of the FLAIR tumor volume is $867 \times 10^{-6} \mathrm{~mm}^{2} / \mathrm{s}$ and $1262 \times 10^{-6} \mathrm{~mm}^{2} / \mathrm{s}$ for the enhancing tumor volume.

Table 2: Predictors of AROC

\begin{tabular}{lcccccc}
\hline \multicolumn{1}{c}{ Predictor } & AROC & SE & $\begin{array}{c}95 \% \\
\text { Lower CL }\end{array}$ & $\begin{array}{c}95 \% \\
\text { Upper CL }\end{array}$ & $\begin{array}{c}\boldsymbol{T} \\
\text { Value }\end{array}$ & $\begin{array}{c}\boldsymbol{P} \\
\text { Value }\end{array}$ \\
\hline$K^{\text {trans }}$ & 0.883 & 0.052 & 0.781 & 0.984 & 7.40 & $<.001$ \\
$K_{\text {ep }}$ & 0.908 & 0.047 & 0.815 & 1.0 & 8.59 & $<.001$ \\
$\mathrm{~V}_{\mathrm{e}}$ & 0.843 & 0.066 & 0.713 & 0.972 & 5.19 & $<.001$ \\
$\mathrm{FL}$ ADC_mean & 0.870 & 0.055 & 0.762 & 0.978 & 6.74 & $<.001$ \\
PG_ADC_mean & 0.820 & 0.066 & 0.691 & 0.949 & 4.86 & $<.001$ \\
$\mathrm{Ve}+\mathrm{FL}$ _ADC_mean & 0.918 & 0.043 & 0.834 & 1.0 & 9.81 & $<.001$ \\
\hline
\end{tabular}

Note:- $C L$ indicates confidence limit.

cating leaky vessels. This finding is consistent with the angiogenesis seen in high-grade tumors caused by neoangiogenic proteins such as vascular endothelial growth factor. Our finding of lower $\mathrm{v}_{\mathrm{e}}$ in high-grade tumors differs from reports in adult glioblastomas, ${ }^{3,24,25}$ likely due to the multiple different high-grade tumor types in children. Jensen et al, ${ }^{26}$ however, found that adult patients with gliomas with lower $\mathrm{v}_{\mathrm{e}}$ in peritu- moral edema had significantly shorter overall survival rates, supporting our hypothesis that lower $\mathrm{v}_{\mathrm{e}}$ corresponds to a higher grade and more aggressive tumor.

Of the ADC histogram metrics we tested, FL_ADC_mean and PG_ ADC_mean showed significant differences between low- and high-grade tumors, with lower ADC corresponding to high-grade tumors. Lower ADC indicates high tumor cellularity and has been associated with shorter survival as well..$^{9-11}$ ADC histogram skewness and kurtosis did not show the same significant differences. Although the number of ADC histograms with bimodal peaks was too small for statistical analysis (6 for $\mathrm{FL}_{-}$ ADC_mean and 4 for PG_ADC_mean), all tumors exhibiting bimodal peaks were high-grade. High-grade tumors often yield bi- 


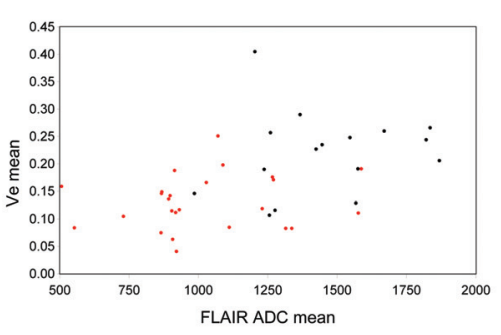

A
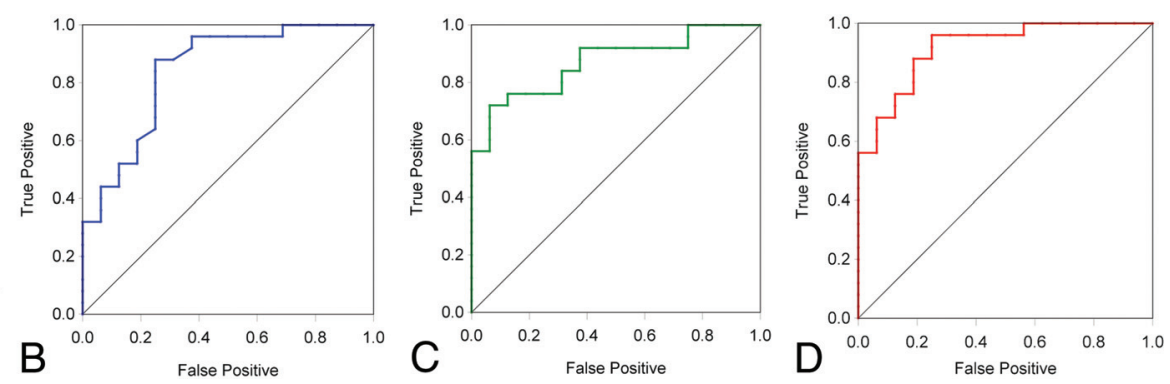

FIG 3. $A$, Scatterplot of $v_{e}$ versus FL_ADC_mean for all 41 cases analyzed. High-grade tumors are shown in red, and low-grade, in black. B, ROC curve for $\mathrm{v}_{\mathrm{e}}(\mathrm{AROC}=0.843)$. $C$, ROC curve for FL_ADC mean $(A R O C=0.870)$. $D, R O C$ curve when $\mathrm{v}_{\mathrm{e}}$ and $\mathrm{FL}$ ADC_mean are combined $(A R O C=0.918)$. Note the improved accuracy shown for the multiparametric predictor, reflected in the increased $\bar{A} A O \bar{C}$.

modal ADC distributions due to the presence of peritumoral edema. ${ }^{9-11}$

We did not detect any group differences among the molecular subtypes of medulloblastoma, which may be due to the small sample size. Recent studies have reported other distinguishing MR imaging features of some of these subtypes, ${ }^{27-29}$ and a larger future sample size may show differences in permeability and ADC histogram metrics among the subtypes. We would postulate that the group 3 tumors, which are ill-defined and have the poorest prognosis, ${ }^{30}$ would have high $K^{\text {trans }}$ and $K_{\text {ep }}$ and low $\mathrm{v}_{\mathrm{e}}$ with low mean ADC histogram values.

Because $\mathrm{v}_{\mathrm{e}}$ is a measure of the extracellular extravascular space and $\mathrm{ADC}$ is also indicative of extracellular space, we tested for a correlation between ADC and $\mathrm{v}_{\mathrm{e}}$. ADC and $\mathrm{v}_{\mathrm{e}}$ were highly correlated across the entire cohort $(P=.001)$. Although a previous study was unable to find voxelwise correlations between $\mathrm{v}_{\mathrm{e}}$ and ADC in adult gliomas, ${ }^{31}$ our findings suggest a link between the mean values in pediatric brain tumors, possibly due to the different tumor types seen in children.

Permeability metrics assess tumor microvasculature, whereas ADC histogram metrics assess tumor cellularity. We hypothesized that these modalities may provide complementary information to assist in tumor grading and tested combinations of permeability and ADC variables as predictors of tumor grade using ROC curves. A combination of $\mathrm{v}_{\mathrm{e}}$ and FL_ADC_mean showed a higher AROC (0.918) compared with $\mathrm{v}_{\mathrm{e}}(0.843)$ or FL_ADC_mean (0.870) alone. Thus, multiparametric analysis may yield the best predictors of tumor grade in pediatric brain tumors.

One limitation of the study is the relatively small number and heterogeneity of tumors studied. Larger numbers of individual tumor types are necessary to validate these findings across all tumor types. The addition of $\mathrm{PET}^{19}$ and MR spectroscopy ${ }^{7,18}$ has also shown promise and may benefit future analyses. Future prospective work could include other multiparametric approaches incorporating ADC histogram and T1 permeability to differentiate molecular and genomic subtypes of pediatric brain tumors.

\section{CONCLUSIONS}

The permeability metrics $K^{\text {trans }}, K_{\text {ep }}$, and $\mathrm{v}_{\mathrm{e}}$ and the ADC histogram metrics FL_ADC_mean and PG_ADC_mean were able to distinguish low- and high-grade pediatric brain tumors with a high degree of accuracy. A multiparametric approach combining permeability and ADC histogram metrics improves the accuracy of pediatric brain tumor grading with high sensitivity and specificity.
Disclosures: Tina Young Poussaint—UNRELATED: Grants/Grants Pending: Pediatric Brain Tumor Consortium Neuroimaging Center, Comments: National Institutes of Health grant with subcontract*; Royalties: Springer Science for a book. *Money paid to the institution.

\section{REFERENCES}

1. Ostrom QT, Gittleman H, Xu J, et al. CBTRUS Statistical Report: Primary Brain and Other Central Nervous System Tumors Diagnosed in the United States in 2009-2013. Neuro Oncol 2016;18:v1v75 CrossRef Medline

2. Falk A, Fahlström M, Rostrup E, et al. Discrimination between glioma grades II and III in suspected low-grade gliomas using dynamic contrast-enhanced and dynamic susceptibility contrast perfusion MR imaging: a histogram analysis approach. Neuroradiology 2014;56:1031-38 CrossRef Medline

3. Li X, Zhu Y, Kang H, et al. Glioma grading by microvascular permeability parameters derived from dynamic contrast-enhanced MRI and intratumoral susceptibility signal on susceptibility weighted imaging. Cancer Imaging 2015;15:4 CrossRef Medline

4. Roberts HC, Roberts TP, Ley S, et al. Quantitative estimation of microvascular permeability in human brain tumors: correlation of dynamic Gd-DTPA-enhanced MR imaging with histopathologic grading. Acad Radiol 2002;9:S151-55 CrossRef Medline

5. Zhang N, Zhang L, Qiu B, et al. Correlation of volume transfer coefficient $\mathbf{K}^{\text {trans }}$ with histopathologic grades of gliomas. JMagn Reson Imaging 2012;36:355-63 CrossRef Medline

6. Vajapeyam S, Stamoulis C, Ricci K, et al. Automated processing of dynamic contrast-enhanced MRI: correlation of advanced pharmacokinetic metrics with tumor grade in pediatric brain tumors. AJNR Am J Neuroradiol 2017;38:170-75 CrossRef Medline

7. Koob M, Girard N, Ghattas B, et al. The diagnostic accuracy of multiparametric MRI to determine pediatric brain tumor grades and types. J Neurooncol 2016;127:345-53 CrossRef Medline

8. Poussaint TY, Rodriguez D. Advanced neuroimaging of pediatric brain tumors: MR diffusion, MR perfusion, and MR spectroscopy. Neuroimaging Clin N Am 2006;16:169-92, ix CrossRef Medline

9. Pope WB, Qiao XJ, Kim HJ, et al. Apparent diffusion coefficient histogram analysis stratifies progression-free and overall survival in patients with recurrent GBM treated with bevacizumab: a multicenter study. J Neurooncol 2012;108:491-98 CrossRef Medline

10. Rahman R, Hamdan A, Zweifler R, et al. Histogram analysis of apparent diffusion coefficient within enhancing and nonenhancing tumor volumes in recurrent glioblastoma patients treated with bevacizumab. J Neurooncol 2014;119:149-58 CrossRef Medline

11. Poussaint TY, Vajapeyam S, Ricci KI, et al. Apparent diffusion coefficient histogram metrics correlate with survival in diffuse intrinsic pontine glioma: a report from the Pediatric Brain Tumor Consortium. Neuro Oncol 2016;18:725-34 CrossRef Medline

12. Tofts PS. Modeling tracer kinetics in dynamic Gd-DTPA MR imaging. J Magn Reson Imaging 1997;7:91-101 CrossRef Medline

13. Weinmann HJ, Laniado M, Mützel W. Pharmacokinetics of GdDTPA/dimeglumine after intravenous injection into healthy volunteers. Physiol Chem Phys Med NMR 1984;16:167-72 Medline 
14. Jenkinson M, Beckmann CF, Behrens TE, et al. FSL. Neuroimage 2012;62:782-90 CrossRef Medline

15. Schindelin J, Arganda-Carreras I, Frise E, et al. Fiji: an open-source platform for biological-image analysis. Nat Methods 2012;9:676-82 CrossRef Medline

16. SAS/STAT 14.1 User's Guide. July 2015. http://support.sas.com/ documentation/cdl/en/statug/68162/HTML/default/viewer.htm\# titlepage.htm. Updated November 2, 2015

17. Morana G, Piccardo A, Tortora D, et al. Grading and outcome prediction of pediatric diffuse astrocytic tumors with diffusion and arterial spin labeling perfusion MRI in comparison with $18 \mathrm{~F}-$ DOPA PET. Eur J Nucl Med Mol Imaging 2017;44:2084-89 CrossRef Medline

18. Tzika AA, Astrakas LG, Zarifi MK, et al. Multiparametric MR assessment of pediatric brain tumors. Neuroradiology 2003;45:1-10 CrossRef Medline

19. Zukotynski KA, Fahey FH, Vajapeyam S, et al. Exploratory evaluation of MR permeability with 18F-FDG PET mapping in pediatric brain tumors: a report from the Pediatric Brain Tumor Consortium. J Nucl Med 2013;54:1237-43 CrossRef Medline

20. Tensaouti F, Ducassou A, Chaltiel L, et al; radiotherapy committee of the French Society for Childhood Cancer (SFCE). Prognostic and predictive values of diffusion and perfusion MRI in paediatric intracranial ependymomas in a large national study. Br J Radiol 2016; 89:20160537 CrossRef Medline

21. Ho CY, Cardinal JS, Kamer AP, et al. Relative cerebral blood volume from dynamic susceptibility contrast perfusion in the grading of pediatric primary brain tumors. Neuroradiology 2015;57:299-306 CrossRef Medline

22. Ho CY, Cardinal JS, Kamer AP, et al. Contrast leakage patterns from dynamic susceptibility contrast perfusion MRI in the grading of primary pediatric brain tumors. AJNR Am J Neuroradiol 2016;37: 544-51 CrossRef Medline

23. Yeom KW, Mitchell LA, Lober RM, et al. Arterial spin-labeled per- fusion of pediatric brain tumors. AJNR Am J Neuroradiol 2014;35: 395-401 CrossRef Medline

24. Abe T, Mizobuchi $\mathrm{Y}$, Nakajima K, et al. Diagnosis of brain tumors using dynamic contrast-enhanced perfusion imaging with a short acquisition time. Springerplus 2015;4:88 CrossRef Medline

25. Choi HS, Kim AH, Ahn SS, et al. Glioma grading capability: comparisons among parameters from dynamic contrast-enhanced MRI and ADC value on DWI. Korean J Radiol 2013;14:487-92 CrossRef Medline

26. Jensen RL, Mumert ML, Gillespie DL, et al. Preoperative dynamic contrast-enhanced MRI correlates with molecular markers of hypoxia and vascularity in specific areas of intratumoral microenvironment and is predictive of patient outcome. Neuro Oncol 2014;16: 280-91 CrossRef Medline

27. Perreault S, Ramaswamy V, Achrol AS, et al. MRI surrogates for molecular subgroups of medulloblastoma. AJNR Am J Neuroradiol 2014;35:1263-69 CrossRef Medline

28. Lastowska M, Jurkiewicz E, Trubicka J, et al. Contrast enhancement pattern predicts poor survival for patients with non-WNT/SHH medulloblastoma tumours. J Neurooncol 2015;123:65-73 CrossRef Medline

29. Yeom KW, Mobley BC, Lober RM, et al. Distinctive MRI features of pediatric medulloblastoma subtypes. AJR Am J Roentgenol 2013; 200:895-903 CrossRef Medline

30. Ramaswamy V, Northcott PA, Taylor MD. FISH and chips: the recipe for improved prognostication and outcomes for children with medulloblastoma. Cancer Genet 2011;204:577-88 CrossRef Medline

31. Mills SJ, Soh C, Rose CJ, et al. Candidate biomarkers of extravascular extracellular space: a direct comparison of apparent diffusion coefficient and dynamic contrast-enhanced MR imaging-derived measurement of the volume of the extravascular extracellular space in glioblastoma multiforme. AJNR Am J Neuroradiol 2010;31: 549-53 CrossRef Medline 